\title{
Vol. 69, No. 31
}

In the report "Alcohol Use and Co-Use of Other Substances Among Pregnant Females Aged 12-44 Years - United States, 2015-2018," on page 1011, errors occurred in the "Race/ Ethnicity" section of Table 1 . In the row for "Black, nonHispanic," the numbers under "Past 30 days binge drinking*" should have read $7.2(4.6-10.9){ }^{\S}$ and in the row for "Other," the numbers under "Past 30 days drinking*" should have read $8.4(4.8-14.4)^{\S}$. 